\title{
TU/e EmonOWEN

\section{Power Scheduling of Fuel Cell Cars in an Islanded Mode Microgrid With Private Driving Patterns}

\section{Citation for published version (APA):}

Alavi, F., van de Wouw, N., \& De Schutter, B. (2020). Power Scheduling of Fuel Cell Cars in an Islanded Mode Microgrid With Private Driving Patterns. IEEE Transactions on Control Systems Technology, 28(4), 1393-1403. [8706997]. https://doi.org/10.1109/TCST.2019.2911491

\section{Document license:}

TAVERNE

DOI:

10.1109/TCST.2019.2911491

Document status and date:

Published: 01/07/2020

\section{Document Version:}

Publisher's PDF, also known as Version of Record (includes final page, issue and volume numbers)

\section{Please check the document version of this publication:}

- A submitted manuscript is the version of the article upon submission and before peer-review. There can be important differences between the submitted version and the official published version of record. People interested in the research are advised to contact the author for the final version of the publication, or visit the $\mathrm{DOI}$ to the publisher's website.

- The final author version and the galley proof are versions of the publication after peer review.

- The final published version features the final layout of the paper including the volume, issue and page numbers.

Link to publication

\section{General rights}

Copyright and moral rights for the publications made accessible in the public portal are retained by the authors and/or other copyright owners and it is a condition of accessing publications that users recognise and abide by the legal requirements associated with these rights.

- Users may download and print one copy of any publication from the public portal for the purpose of private study or research.

- You may not further distribute the material or use it for any profit-making activity or commercial gain

- You may freely distribute the URL identifying the publication in the public portal.

If the publication is distributed under the terms of Article 25fa of the Dutch Copyright Act, indicated by the "Taverne" license above, please follow below link for the End User Agreement:

www.tue.nl/taverne

Take down policy

If you believe that this document breaches copyright please contact us at:

openaccess@tue.nl

providing details and we will investigate your claim. 


\title{
Power Scheduling of Fuel Cell Cars in an Islanded Mode Microgrid With Private Driving Patterns
}

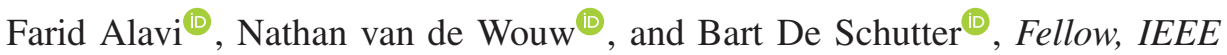

\begin{abstract}
A microgrid in the islanded mode is considered where a fleet of fuel cell cars is used as a distributed power generation system. The objective of the proposed control system is to minimize the operational cost of the system, subject to the physical and operational constraints of the system. In order to deal with uncertainty in the prediction of the microgrid's load, two model predictive control methods, a min-max (MM) approach and disturbance feedback MM approach, are proposed. We develop three distributed control algorithms and we show that by using these algorithms, the driving patterns of the fuel cell cars can be kept private. In other words, no privacy sensitive data on the usage of the cars are collected by a central control agent. Numerical case studies are presented to demonstrate the excellent performance of the proposed control methods.
\end{abstract}

Index Terms-Decentralized control, distributed power generation, fuel cells, smart grids.

\section{INTRODUCTION}

$\mathbf{S}$ EVERAL methods of microelectrical power generation have been developed in recent years. Examples of such microgenerators are solar photovoltaic cells, microwind turbines, and combined heat and power units. By connecting several microgenerators to the low-voltage electrical connection of an area and equipping each generator with communication facilities, a microgrid can be constructed that is able to operate in two different modes. In the first operational mode, a gridconnected mode microgrid, the microgrid is connected to the main power grid, while in the second mode, an islanded mode microgrid, there is not any electrical connection between the microgrid and any other power grids [1]. In this paper, we focus on the power scheduling of an islanded mode microgrid.

In recent years, fuel cell cars are being produced by several companies in the world. A fuel cell car is equipped with

Manuscript received July 12, 2018; revised January 22, 2019; accepted March 25, 2019. Date of publication May 6, 2019; date of current version June 11, 2020. Manuscript received in final form April 12, 2019. This work was supported by the NWO-URSES Project Car as Power Plant financed by the Netherlands Organization for Scientific Research (NWO). Recommended by Associate Editor W. Zhang. (Corresponding author: Farid Alavi.)

F. Alavi and B. De Schutter are with the Delft Center for Systems and Control, Delft University of Technology, 2628 CD Delft, The Netherlands (e-mail: f.alav@tudelft.nl; b.deschutter@tudelft.nl).

N. van de Wouw is with the Delft Center for Systems and Control, Delft University of Technology, 2628 CD Delft, The Netherlands, also with the Department of Mechanical Engineering, Eindhoven University of Technology, 5600 MB Eindhoven, The Netherlands, and also with the Department of Civil, Environmental and Geo-Engineering, University of Minnesota, Minneapolis, MN 55455 USA (e-mail: n.vandewouw@tue.nl).

Color versions of one or more of the figures in this article are available online at http://ieeexplore.ieee.org.

Digital Object Identifier 10.1109/TCST.2019.2911491 all the necessary devices to generate electricity from the chemical energy of hydrogen. As a result, a fleet of fuel cell cars can be used as a distributed power generation system inside a microgrid. The feasibility of such microgrids, based on fuel cell cars, is studied in [2] and [3]. We refer the reader to [2] for a justification about rationality of using fuel cell cars as the source of electrical power generation in the future. In this paper, we consider the concept of car as power plant [4], in which a fleet of fuel cell cars is used to generate power inside a microgrid, where the electrical power generation by fuel cells has a higher efficiency compared to the conventional power plants that are using fossil fuels. We consider a microgrid in the islanded mode, where the power generation of renewable energy sources (RES) inside the microgrid is not curtailed for the sake of power balance. Instead, in order to guarantee the power balance condition, the power generation of fuel cell cars is controlled. We assume that each car is equipped with a controller that is able to set the power generation profile of the car's fuel cell to a desirable level, see [5].

Different levels of control can be used in order to maintain the power balance within a microgrid in the islanded mode. Traditionally, three control levels exist in power systems, namely, primary, secondary, and tertiary controls. In primary and secondary controls [6]-[8], the objective of the control system is to keep the voltage and frequency of the power system at a specific value. In tertiary control [9], set points for the amount of power generation are determined for each generator. In this level, the objective is to minimize the operational cost of the system while considering the power balance condition and the system constraints, such as the maximum power generation of each generator. Model predictive control (MPC) is commonly used in tertiary control or power scheduling [10][12]. In this paper, we consider tertiary control, or the power scheduling problem of a microgrid using MPC method.

The presence of uncertainty in microgrids with RES necessitates the use of robust control methods for the power scheduling task. A min-max (MM) MPC approach is proposed in [13], where the energy balance of the microgrid is satisfied via the curtailment of the RES generation. Stochastic optimization [14], [15] and scenario-based optimization [16], [17] are among the other power scheduling methods developed to deal with the uncertainty in the prediction of the load.

In this paper, an MM method and a disturbance feedback (DF) MM method are developed for a microgrid with fuel cell cars operating in islanded mode. In our conference paper [18], we have shown that centralized versions of both of 
TABLE I

DESCRIPTION OF THE NotATIONS USED IN THIS PAPER

\begin{tabular}{|l|l|}
\hline Notation & Description \\
\hline$x_{\mathrm{f}}$ & Fuel level of cars \\
$u_{\mathrm{f}}$ & Power generation of fuel cell stack in vehicle to grid mode \\
$T_{\mathrm{S}}$ & Sampling time \\
$\alpha_{\mathrm{f}}, \beta_{\mathrm{f}}$ & Two constant parameters related to the specification of \\
& fuel cell stacks \\
$R_{\mathrm{f}}$ & Refilling rate of fuel cell cars \\
$h_{\mathrm{f}}$ & Consumed fuel due to transportation \\
$s_{\mathrm{f}}$ & Switching signal of fuel cell stacks in vehicle to grid mode \\
$s_{\mathrm{r}}$ & A binary variable indicating the refilling process of cars \\
$\lambda_{\mathrm{f}}$ & A binary variable indicating the transportation mode of cars \\
$w$ & Uncertainty in the power generation of fuel cell stacks \\
$P_{\mathrm{d}}$ & Residual load of the microgrid \\
\hline
\end{tabular}

the proposed methods are able to guarantee the power balance of the microgrid. However, the DF MM method increases the system performance compared to the MM method, by reducing the conservatism in the operation of the system. A centralized control system based on the MM method or the DF MM method requires information on the driving pattern of all the cars for its operation. Here, the driving pattern is defined as the information about the departure time, the driving distance, and the time of return to the microgrid for any fuel cell car. We consider the driving pattern of a car as private information and, as a result, design a control system that requires the share of driving patterns violates privacy rights of car owners. In this paper, these privacy rights are considered as a key requirement in the design of the control system.

The main contribution of our work is that we develop three distributed DF MM methods for power scheduling of a microgrid with fuel cell cars that are shown to be more efficient than existing methods from the literature. In addition, the power scheduling problem is formulated while considering specific features of the system, such as the driving patterns of the cars, the refilling process of the cars, and the privacy of the fuel cell car owners.

This paper extends our early results presented in the conference paper [18] by developing the three distributed control approaches using a refined formulation of the MM method and the DF MM method and more extensive case studies of the system. The performance of each distributed control method is demonstrated via case studies with different sizes of the microgrid. It is shown that for a large number of vehicles in the microgrid, the performance of the distributed control methods is close to a model predictive controller with centralized architecture, while the computational burden in the distributed architectures is reduced compared to the centralized architecture.

The rest of this paper is organized as follows. The problem is formulated in Section II. MPC of the system with a centralized architecture is introduced in Section III. The three distributed control methods are developed in Section IV and the results of illustrative case studies are presented in Section V. A summary of the notations used in this paper is presented in Table I.

\section{Problem Formulation}

\section{A. System Description}

We consider a problem setting in which a fleet of fuel cell cars is acting as the power generation source of a microgrid. Besides the fuel cell cars, wind turbines, solar photovoltaic cells, and diesel generators are the other sources of power generation. It is assumed that the microgrid is operated in the islanded mode and there is no control over the load of the microgrid. Therefore, the power balance should be maintained via the control of generation units. The goal of the microgrid's operator is, first, to allow the renewable energy sources to generate as much power as possible based on the weather condition and, second, to penalize the use of the diesel generators. With these requirements, the power balance of microgrid should be realized by a suitable power scheduling of the fuel cell cars.

The objective of the control method proposed in this paper is similar to a traditional tertiary control algorithm in the sense that the objective of the control algorithm is also to minimize the operational cost of the system while considering system constraints. However, a fleet of fuel cell cars in an islanded mode microgrid has some unique features that further challenge the controller design. In particular, the fuel cell cars are used both for power generation and transportation, the amount of fuel is limited, and refilling the cars takes time. These unique features necessitate a new design for the control system. The objective of the control system is to determine a power generation profile for each fuel cell car in such a way that the power balance of the microgrid is satisfied. In addition, the central control system should exclude those cars from the power generation units of which the owners intend to use them for transportation.

From an energy management point of view, the functioning of primary and secondary control levels affects the power generation of the generator, and as a result, the generated power typically deviates from the scheduled power generation profile. Here, we assume that such control levels exist in each power generation unit, and as a result, the scheduled and the actual power generation are not necessarily equal to each other. The mismatch between the scheduled and the actual power generation is considered as an uncertain disturbance in the model of the system. With this abstraction of the influence of the primary and secondary control levels, we focus on the power scheduling problem on the tertiary level.

In the scenario considered here, the communication between different agents of a system is considered to be ideal. Therefore, no delay, packet loss, or cost is considered in the communication process. All the cars are connected to the microgrid whenever they are not used for the transportation or refueling. In addition, each car is equipped with a computer that can operate the fuel cell of the car and exchange information with the central control system.

\section{B. Mixed Logical Dynamical Model of the System}

One of the most significant features of the considered microgrid is that the fuel cell cars are used for two purposes: transportation and power generation. In addition, the fuel 
stored in each car is limited and refueling each car takes time. In this section, with inspiration from [19] and [18], a mixed logical dynamical model is developed for the system.

We consider the remaining fuel in each car as a system state. Therefore, the system states can change both in the transportation and power generation mode. The transportation mode of each car is determined by the driving pattern of the car owner. We assume that by studying the behavior of each driver, it is possible to determine the driving pattern of each driver for a finite horizon in the future from time step $k$ to $k+N_{p}$, where $N_{p}$ is the prediction horizon. By defining $\mathcal{P}=\left\{0,1, \ldots, N_{p}-1\right\}$ and $\mathcal{I}=\left\{1, \ldots, N_{\text {veh }}\right\}$ with $N_{\text {veh }}$ the total number of cars in the system, we can model the availability of a car $i \in \mathcal{I}$ for power generation at time steps $k+j$, for all $j \in \mathcal{P}$, with a sequence of binary variables $\lambda_{f, i}(k+j)$ for $i \in \mathcal{I}$ and $j \in \mathcal{P}$ : if fuel cell car $i$ is in the transportation mode at time step $k+j$, then $\lambda_{f, i}(k+j)$ is equal to 1 and it is 0 otherwise. In addition, we assume that if a car is used for transportation from time step $k$ to time step $k+j$, the amount of fuel that is used for that trip is predictable and described by $h_{f, i}(k+j)$. Therefore, we can assume that if a car is in the transportation mode, the fuel level remains the same, and at the return time step $k+j$, it will be reduced with the amount $h_{f, i}(k+j)$. We also consider a specific mode for refilling a car where the fuel level is increased by $R_{f, i}$ at each time step. Note that as a microgrid can span a wide geographical region, and the fuel cell cars can be parked at different locations, we assume that hydrogen would be available only at a few spots. This is the main reason that we need to refill the hydrogen tank of fuel cell cars.

By considering the relation between the fuel consumption and the net power generation of a fuel cell [20], we can derive the following equation for the evolution of the fuel level of car $i \in \mathcal{I}$ in the power generation mode:

$$
x_{f, i}(k+1)=x_{f, i}(k)-\left(\alpha_{f, i} u_{f, i}^{*}(k)+\beta_{f, i}\right) T_{\mathrm{s}}
$$

where $\alpha_{f, i}$ and $\beta_{f, i}$ are parameters related to the fuel cell $i$ and $T_{\mathrm{s}}$ is the sampling time interval. The actual power generation of fuel cell $i$ at time step $k$ is indicated by $u_{f, i}^{*}(k)$. Considering that the primary and secondary controllers may induce actual power generation levels that deviate from the scheduled level, we have

$$
u_{f, i}^{*}(k)=u_{f, i}(k)+w_{i}(k) \quad \forall k
$$

where $u_{f, i}(k)$ indicates the scheduled power generation of fuel cell $i \in \mathcal{I}$ at time step $k$ and $w_{i}(k)$ is an unknown time-varying deviation from the scheduled value.

By gathering all the operational modes of fuel cell car $i \in \mathcal{I}$, the following piecewise affine model describes the system dynamics:

$$
\begin{aligned}
& x_{f, i}(k+1) \\
& = \begin{cases}x_{f, i}(k)+R_{f, i} & \text { refilling } \\
x_{f, i}(k) & \text { no generation and } \\
& \text { transportation } \\
x_{f, i}(k)-\left(\alpha_{f, i} u_{f, i}^{*}(k)+\beta_{f, i}\right) T_{S} & \text { generation } \\
x_{f, i}(k)-h_{f, i}(k) & \text { return. }\end{cases}
\end{aligned}
$$

The motivation behind this model is discussed in more detail in [19]. Note that a car in the driving mode influences the control system only by changing the remaining fuel in its tank. As the car is not used for the power generation until it returns to the microgrid's area, only the amount of remaining fuel at the arrival time is important. Therefore, the loss of communication when the car is out of range is not a problem.

To indicate the operational mode of fuel cell car $i \in \mathcal{I}$, we use two binary variables $s_{f, i}$ and $s_{r, i}$. The refilling mode at time step $k$ is indicated by $s_{r, i}(k)=1$; in other modes, $s_{r, i}(k)=0$. The power generation mode corresponds to $s_{f, i}(k)=1$ and, if $s_{f, i}(k)=0$, the fuel cell $i$ is turned off. It is assumed that while a fuel cell car is in the driving mode, it can neither generate power for the microgrid nor be refilled. These constraints can be represented by

$$
\begin{aligned}
& \text { if } \lambda_{f, i}(k)=1 \quad \text { then } s_{r, i}(k)=0 \\
& \text { if } \lambda_{f, i}(k)=1 \quad \text { then } s_{f, i}(k)=0 .
\end{aligned}
$$

We assume that if a fuel cell is being refilled, it cannot generate power. Other constraints in the operation of a fuel cell include the maximum level of power generation and the maximum fuel level. Moreover, a fuel cell can generate power only when the fuel level of the car is above a certain minimum level. These constraints can be represented as follows:

$$
\begin{aligned}
\text { if } s_{r, i}(k) & =1 \text { then } s_{f, i}(k)=0 \\
0 & \leq u_{f, i}(k) \leq \bar{u}_{f, i} \\
\underline{x}_{f, i} s_{f, i}(k) & \leq x_{f, i}(k) \leq \bar{x}_{f, i} .
\end{aligned}
$$

The equivalent mixed logical dynamical model [21] of the system in (3) with the constraints as explicated above is given by

$$
\begin{aligned}
x(k+1)= & x(k)+B_{1}(w(k)) u(k) \\
& +B_{3}(k) z(k)+B_{4}(k) \\
E_{3} z(k) \leq & E_{1} u(k)+E_{4} x(k)+E_{5}(k)
\end{aligned}
$$

where the vectors $x, u$, and $z$ are defined in (5). Note that, for the sake of compactness, the time step $k$ is dropped in the following equations. We define:

$$
\begin{aligned}
& x=\left[\begin{array}{lll}
x_{f, 1} & \ldots & x_{f, N_{\mathrm{veh}}}
\end{array}\right]^{T} \\
& u=\left[\begin{array}{lllllll}
u_{f, 1} & s_{r, 1} & s_{f, 1} & \ldots & u_{f, N_{\mathrm{veh}}} & s_{r, N_{\mathrm{veh}}} & s_{f, N_{\mathrm{veh}}}
\end{array}\right]^{T} \\
& z=\left[\begin{array}{lll}
z_{f, 1} & \ldots & z_{f, N_{\mathrm{veh}}}
\end{array}\right]^{T} .
\end{aligned}
$$

The continuous auxiliary variables $z_{f, i}$ are defined as $z_{f, i}(k) \triangleq$ $s_{f, i}(k) u_{f, i}(k)$, for all $i \in \mathcal{I}$ and $k$. With defining $\operatorname{diag}\{$.$\} as a$ block diagonal matrix with the arguments as diagonal blocks, we can define matrices $B_{1}(k), B_{3}(k)$, and $B_{4}(k)$ as follows:

$$
\begin{aligned}
& B_{1}(k)=\operatorname{diag}\left\{b_{1}^{1}(k), \ldots, b_{1}^{N_{\text {veh }}}(k)\right\} \\
& B_{3}(k)=\operatorname{diag}\left\{b_{3}^{1}(k), \ldots, b_{3}^{N_{\text {veh }}}(k)\right\} \\
& B_{4}(k)=\left[\begin{array}{lll}
-\lambda_{f, 1}(k) h_{f, 1}(k) & \ldots & -\lambda_{f, N_{\text {veh }}}(k) h_{f, N_{\text {veh }}}(k)
\end{array}\right]^{T}
\end{aligned}
$$

where $b_{1}^{i}(k)=\left[0 R_{f, i}\left(1-\lambda_{f, i}(k)\right) T_{s}\left(\beta_{f, i}+\alpha_{f, i} w_{i}(k)\right)\right]$ and $b_{3}^{i}=\left(1-\lambda_{f, i}(k)\right) T_{s} \alpha_{f, i}$, for $i \in \mathcal{I}$. 


\section{Centralized Robust Control Architecture}

The power scheduling problem of the considered microgrid can be solved by designing a central control system for the whole microgrid. In this scenario, a control center gathers all the relevant information from the devices in the microgrid, determines a power scheduling profile for each fuel cell car, and sends these profiles to each car. To determine the power scheduling profiles, the operational cost of the system over a finite horizon is minimized subject to the system constraints. In this section, two centralized MPC approaches are developed by considering the specific features of the system.

\section{A. MM Control Method}

In the framework of MPC, the operational cost of the system is minimized over a finite time window with respect to the system constraints. The model for the system developed in Section II-B contains an uncertain variable, $w$, see (2), and to deal with this uncertainty, an MM control method is proposed in [19]. In the MM control method, the operational cost of the system is minimized for the worst case realization of the uncertainty. In addition, by satisfying the system constraints for the worst case uncertainty, the MM control method guarantees the satisfaction of the constraints for any realization of the uncertainty.

We can define the following cost function for the system [19]:

$$
\begin{gathered}
J(k)=\sum_{j \in \mathcal{P}} \sum_{i \in \mathcal{I}}\left[W_{p, i} u_{f, i}^{2}(k+j)+W_{s, i}\left|\Delta s_{f, i}(k+j)\right|\right. \\
\left.+C_{\mathrm{e}}(k+j) w_{i}(k+j)\right]
\end{gathered}
$$

where $\Delta s_{f, i}(k+j)$ is defined as $s_{f, i}(k+j)-s_{f, i}(k+j-1)$. The two parameters, $W_{p, i}$ and $W_{s, i}$, determine the cost of power generation and the cost of switching the operational mode of a fuel cell. The value of $w_{i}(k+j)$ indicates the deviation from the scheduled power generation of fuel cell $i \in \mathcal{I}$ at time step $k+j$. This deviation is the result of an effort for stabilizing the microgrid. In order to encourage the fuel cell cars to stabilize the microgrid, we assume that each fuel cell $i$ for $i \in \mathcal{I}$ gets a reward equal to $C_{\mathrm{e}}(k+j) w_{i}(k+j)$ at time step $k+j$ for the willingness to deviate its actual power generation from the scheduled one by the amount of $w_{i}(k+j)$. This reward for fuel cell cars is an additional cost for the microgrid operator.

Note that the quadratic part of the cost function (6), i.e., $W_{p, i} u_{f, i}^{2}(k)$, is $W_{p, i} u_{f, i}^{2}(k)$, is mainly the result of the degradation of the fuel cells due to the defined in a quadratic way because by increasing the net power generation of a fuel cell, some auxiliary devices such as the fuel cell cooling system and the air compressor will be activated, which result in ever-increasing operational cost. On the other hand, the deviation $w_{i}$ is penalized by a linear factor because it is related to the electricity market of the primary and secondary control levels. As a result, each unit of electrical energy has a predefined price at time step $k$, i.e., $C_{\mathrm{e}}(k)$, and the cost function related to $w_{i}$ becomes linear.
By defining

$$
\tilde{w}_{i}(k)=\left[\begin{array}{lll}
w_{i}(k) & \ldots & w_{i}\left(k+N_{p}-1\right)
\end{array}\right]^{T}
$$

the operational cost of the system can be rewritten as

$$
\begin{gathered}
J(k)=\sum_{i \in \mathcal{I}}\left(\tilde{V}_{i}^{T}(k) W_{q, i}(k) \tilde{V}_{i}(k)+W_{v, i}(k) \tilde{V}_{i}(k)\right. \\
\left.+W_{d, i}(k) \tilde{w}_{i}(k)\right)
\end{gathered}
$$

where $W_{q, i}, W_{v, i}$, and $W_{d, i}$ can be determined for all $i \in \mathcal{I}$ based on the values of $W_{p, i}, W_{s, i}$, and $C_{\mathrm{e}}$, respectively. The vector of optimization variables, $\tilde{V}(k)$, is defined as

$$
\tilde{V}(k)=\left[\begin{array}{lll}
\tilde{V}_{1}^{T}(k) & \ldots & \tilde{V}_{N_{\text {veh }}}^{T}(k)
\end{array}\right]^{T}
$$

where $\tilde{V}_{i}(k)$ is the vector of optimization variables related to fuel cell $i \in \mathcal{I}$

$$
\tilde{V}_{i}(k)=\left[\begin{array}{ll}
\tilde{u}_{i}^{T}(k) & \tilde{z}_{i}^{T}(k)
\end{array}\right]^{T} .
$$

The vectors $\tilde{u}_{i}$ and $\tilde{z}_{i}$ in (9) are the stacked version of the following variables over time steps $k$ to $k+N_{p}-1$ :

$$
\begin{aligned}
u_{i}(k) & =\left[\begin{array}{lll}
u_{f, i}(k) & s_{r, i}(k) & s_{f, i}(k)
\end{array}\right]^{T} \\
z_{i}(k) & =s_{f, i}(k) u_{f, i}(k) .
\end{aligned}
$$

By extending the system constraints in (4) to all the time steps in the prediction window, we can determine the matrices $G_{1, i}, G_{2, i}$, and $G_{3, i}$ such that the following inequalities describe the system constraints in the whole prediction window:

$$
G_{1, i}(\tilde{w}(k)) \tilde{V}_{i}(k) \leq G_{2, i}(k)+G_{3, i}(k) x_{i}(k) \quad \forall i \in \mathcal{I} .
$$

The power balance condition is

$$
\sum_{i \in \mathcal{I}} u_{f, i}(k+j)=P_{d}(k+j) \quad \forall j \in \mathcal{P}
$$

where $P_{d}(k+j)$ is the residual load of the microgrid at time step $k+j$, i.e., the microgrid's load minus the generated power by the renewable energy sources.

In the MM approach, the aim is to minimize the operational cost of the system for the worst case uncertainty, while the system constraints are satisfied for any realization of the uncertainty. Therefore, the following optimization problem should be solved at each time step $k$ :

$$
\begin{gathered}
\min _{\left\{\tilde{V}_{i}(k)\right\}_{i \in \mathcal{I}}} \max _{\left\{\tilde{w}_{i}(k)\right\}_{i \in \mathcal{I}}} \sum_{i \in \mathcal{I}}\left(\tilde{V}_{i}^{T}(k) W_{q, i}(k) \tilde{V}_{i}(k)+W_{v, i}(k) \tilde{V}_{i}(k)\right. \\
\left.\quad+W_{d, i}(k) \tilde{w}_{i}(k)\right) \\
\text { s.t. (10), (11), for all } \tilde{w}_{i} \text { where } i \in \mathcal{I} .
\end{gathered}
$$

We assume that the uncertainties are always realized within given bounds as follows:

$$
\underline{w}_{i} \leq w_{i}(k) \leq \bar{w}_{i} \text {, for all } i \in \mathcal{I} \text { and } k .
$$

The source of uncertainty is the errors in the prediction of the residual load, which contain the errors in the prediction of RES power generation and the microgrid's load. An extensive review on different methods of forecasting the load is presented in [22] and based on this reference, a common 
forecasting method can be written in the following form of $p(t)=f(t)+v(t)$, where $p(t)$ is the predicted load, $f(t)$ is a predetermined function, and $v(t)$ is a white random noise at time $t$. Considering that any white noise signal has a finite variance, it is possible to determine some approximate boundaries on the realization of the noise. Even though there remains a small probability that the realization of the noise is outside the determined bounds, by selecting a large enough boundary, we can neglect the probability that the realization of the noise is outside of the determined boundaries. Because the uncertain variables $w_{i}(k)$ for all $i \in \mathcal{I}$ are proportionally related to the error in the total load of the microgrid, we can define a minimum and a maximum level for the realization of each $w_{i}(k)$.

As a result of (13), we can define the set $\mathcal{W}$ such that

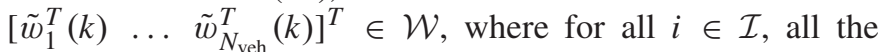
elements of $\tilde{w}_{i}(k)$ comply with the inequalities in (13).

By following the approach mentioned in [18, Lemma 1], we can conclude that by using assumption (13), the following inequalities guarantee that (10) is satisfied for any realization of $\tilde{w}(k) \in \mathcal{W}$ :

$$
\begin{gathered}
G_{1, i}\left(\hat{w}_{1, i}(k)\right) \tilde{V}_{i}(k) \leq G_{2, i}(k)+G_{3, i}(k) x_{i}(k) \quad \forall i \in \mathcal{I} \\
\vdots \\
G_{1, i}\left(\hat{w}_{N, i}(k)\right) \tilde{V}_{i}(k) \leq G_{2, i}(k)+G_{3, i}(k) x_{i}(k) \quad \forall i \in \mathcal{I}
\end{gathered}
$$

where $\hat{w}_{1, i}(k), \ldots, \hat{w}_{N, i}(k)$, with $N=2^{N_{p}}$, are defined as

$$
\begin{aligned}
& \hat{w}_{1, i}(k)=\left[\begin{array}{llll}
\underline{w}_{i}(k) & \underline{w}_{i}(k+1) & \ldots & \underline{w}_{i}\left(k+N_{p}-1\right)
\end{array}\right]^{T} \\
& \hat{w}_{2, i}(k)=\left[\begin{array}{llll}
\underline{w}_{i}(k) & \underline{w}_{i}(k+1) & \ldots & \bar{w}_{i}\left(k+N_{p}-1\right)
\end{array}\right]^{T} \\
& \quad \vdots \\
& \hat{w}_{N, i}(k)=\left[\begin{array}{llll}
\bar{w}_{i}(k) & \bar{w}_{i}(k+1) & \ldots & \bar{w}_{i}\left(k+N_{p}-1\right)
\end{array}\right]^{T} .
\end{aligned}
$$

Therefore, the optimization problem (12) can be rewritten as

$$
\begin{aligned}
\min _{\tilde{V}_{i}(k), i \in \mathcal{I}} \max _{p \in\{1, \ldots, N\}} \sum_{i \in \mathcal{I}}\left(\tilde{V}_{i}^{T}(k) W_{q, i}(k) \tilde{V}_{i}(k)\right. \\
\\
\left.+W_{v, i}(k) \tilde{V}_{i}(k)+W_{d, i}(k) \hat{w}_{p, i}(k)\right)
\end{aligned}
$$$$
\text { s.t. (14) and (11). }
$$

The problem (15) is a mixed integer quadratic programming (MIQP) problem and can be solved by a standard solver such as Gurobi [23].

\section{B. DF MM Control Method}

The MM control method of Section III-A guarantees that the system constraints are satisfied for any realization of the disturbance. However, the level of conservatism in the MM method may be considerable. In [18], a DF control method is developed that yields a better performance when a control law is defined for the power generation of each fuel cell car as follows:

$$
u_{f, i}(k+j)=v_{f, i}(k+j)+K_{f, i}(k+j) w_{i}(k+j-1)
$$

where $v_{f, i}(k+j)$ and $K_{f, i}(k+j)$ are, respectively, the deterministic part of the scheduled power generation and the
DF gain for fuel cell car $i \in \mathcal{I}$ at time step $k+j$. The value of $w_{i}(k+j-1)$ is unknown before time step $k+j$ but after that, it can be determined by subtracting the actual power generation, $u_{f, i}^{*}(k+j-1)$, from the scheduled power generation, $u_{\mathrm{f}, i}(k+j-1)$. Note that in the DF method, the scheduled power generation, $u_{\mathrm{f}, i}(k+j)$, at time step $k+j$ for $j \geq 1$ consists of two parts: a deterministic part, i.e., $v_{\mathrm{f}, i}(k+j)$, and an unknown part, i.e., $K_{\mathrm{f}, i} w_{i}(k+j-1)$.

It can be shown that by defining the matrices $G_{1, i}^{\mathrm{DF}}, G_{2, i}^{\mathrm{DF}}$, and $G_{3, i}^{\mathrm{DF}}$ in an appropriate way, the system constraints (10) can be expressed as

$$
\begin{gathered}
G_{1, i}^{\mathrm{DF}}\left(\hat{w}_{1, i}(k)\right) \tilde{V}_{i}^{\mathrm{DF}}(k) \leq G_{2, i}^{\mathrm{DF}}(k)+G_{3, i}^{\mathrm{DF}}(k) x_{i}(k) \quad \forall i \in \mathcal{I} \\
\vdots \\
G_{1, i}^{\mathrm{DF}}\left(\hat{w}_{N, i}(k)\right) \tilde{V}_{i}^{\mathrm{DF}}(k) \leq G_{2, i}^{\mathrm{DF}}(k)+G_{3, i}^{\mathrm{DF}}(k) x_{i}(k) \quad \forall i \in \mathcal{I}
\end{gathered}
$$

where

$$
\tilde{V}_{i}^{\mathrm{DF}}(k)=\left[\begin{array}{lll}
\tilde{v}_{i}^{T}(k) & \tilde{K}_{f, i}^{T}(k) & \tilde{z}_{i}^{T}(k)
\end{array}\right]^{T} .
$$

The power balance constraint in the DF MM method can be expressed as

$$
\left\{\begin{array}{l}
\sum_{i \in \mathcal{I}} u_{f, i}(k)=P_{d}(k) \\
\sum_{i \in \mathcal{I}} v_{f, i}(k+j)=P_{d}(k+j), \quad j \in\left\{1, \ldots, N_{p}-1\right\} .
\end{array}\right.
$$

The optimization problem of the model predictive controller at time step $k$ can be written as

$$
\begin{aligned}
\min _{\tilde{V}_{i}^{\mathrm{DF}}(k), i \in \mathcal{I}} \max _{p \in\{1, \ldots, N\}} \sum_{i \in \mathcal{I}}\left(\left(\tilde{V}_{i}^{\mathrm{DF}}\right)^{T}(k) W_{q, i}^{\mathrm{DF}}(k) \tilde{V}_{i}^{\mathrm{DF}}(k)\right. \\
\left.+W_{v, i}^{\mathrm{DF}}(k) \tilde{V}_{i}^{\mathrm{DF}}(k)+W_{d, i}^{\mathrm{DF}}(k) \hat{w}_{p, i}(k)\right)
\end{aligned}
$$

s.t. (17) and (18).

where $W_{q, i}^{\mathrm{DF}}(k), W_{v, i}^{\mathrm{DF}}(k)$, and $W_{d, i}^{\mathrm{DF}}(k)$ are defined based on the cost function (6). Any MIQP standard solver can be used in order to solve the optimization problem (19).

The MM and the DF MM methods are able to determine a power schedule for the fuel cell cars such that the system constraints are satisfied. In the DF MM approach, a feedback law on the future disturbances prevents the expansion of the possible state trajectories inside the predicted period, and as a result, the level of conservatism is lower compared to the MM method [18]. However, in order to implement these methods, the driving patterns of all the fuel cell cars should be shared with a central controller. To increase the privacy level of the car owners, three distributed control methods are developed in Section IV.

\section{Distributed Robust Control Architecture}

In this section, three distributed control strategies based on dual decomposition, alternating direction method of multipliers (ADMM), and proximal ADMM (PADMM) are developed in order to support the MM and the DF MM approaches in a distributed fashion. In the developed methods, the driving patterns of the cars are kept private, i.e., there is no need to 
share the information about the departure or arrival time of the cars with any other agent.

The generic distributed control strategies employed in this section were originally developed for convex programming problems [24]-[26]. The presence of binary variables makes the optimization problems (15) and (19) nonconvex and, hence, the developed algorithms might not converge at all or converge to a nonoptimal point. The lack of convergence in the power scheduling process will result in an imbalance in the power generation and usage in the microgrid. However, the use of some diesel generators as backup generation units and some batteries to store energy can still guarantee the power balance condition of the microgrid. To decrease the usage of fossil fuels or batteries, we assume that the correction of power scheduling using backup generation and storage units is much more expensive compared to the generated power of a fuel cell.

The developed methods are based on some iterations, i.e., an information exchange process between a coordinator and the cars. At the end of each iteration, the control system reaches a specific power schedule. If the power balance is satisfied or a maximum number of iterations is reached, the loop of iterations is terminated by the coordinator and the fuel cell cars execute the last power schedule; otherwise, the coordinator starts a new iteration. The backup generation and storage units are used when the loop of iterations is terminated while the power balance condition is not reached.

\section{A. Dual Decomposition Method}

In the dual decomposition approach, a new optimization problem, called the dual problem, is constructed based on the original problem, i.e., the primal problem, e.g., (15) or (19). In some cases, the structure of the dual problem allows us to solve it in a distributed fashion. As will be explained below, the dual problems of (15) and (19) can be separated between all the cars.

In the dual decomposition method, the dual problem is solved in a distributed fashion. Because the optimization problems are not convex, the optimum value of the dual problem might be smaller than that of the primal problem. In other words, there might be a duality gap [24]. The presence of a duality gap means that the power scheduling process has not reached to the balance condition yet; in this case, the backup generation and storage units will be used to guarantee the power balance condition. Note that by design, the control algorithms tend to maintain the power balance using only the fuel cell cars. The backup generation and storage units are used only when the controller fails to maintain the power balance using only fuel cell cars.

The MM problem (15) consists of $N$ MIQP problems, where the $p$ th MIQP (primal) problem is formulated as

$$
\begin{gathered}
\mathrm{P}: \min _{\tilde{V}_{i}(k), i \in \mathcal{I}} \sum_{i \in \mathcal{I}}\left(\tilde{V}_{i}^{T}(k) W_{q, i}(k) \tilde{V}_{i}(k)+W_{v, i}(k) \tilde{V}_{i}(k)\right. \\
\left.\quad+W_{d, i}(k) \hat{w}_{p, i}(k)\right)
\end{gathered}
$$

and its dual problem can be written as

$$
\begin{aligned}
\mathrm{D}: \max _{\lambda(k) \in \mathbb{R}^{N_{p}}} \min _{\tilde{V}_{i}(k), i \in \mathcal{I}} & \sum_{i \in \mathcal{I}}\left(\tilde{V}_{i}^{T}(k) W_{q, i}(k) \tilde{V}_{i}(k)\right. \\
& \left.+W_{v, i}(k) \tilde{V}_{i}(k)+W_{d, i}(k) \hat{w}_{p, i}(k)\right) \\
& +\lambda^{T}(k)\left(\tilde{u}_{\mathrm{t}}(k)-\tilde{P}_{d}(k)\right)
\end{aligned}
$$$$
\text { s.t. (14) }
$$

where $\tilde{u}_{\mathrm{t}}(k)$ and $\tilde{P}_{d}(k)$ are the stacked versions of $u_{\mathrm{t}}$ and $P_{d}$ over time steps $k$ to $k+N_{p}$, that is,

$$
\begin{gathered}
\tilde{u}_{\mathrm{t}}(k)=\left[\begin{array}{lll}
u_{\mathrm{t}}(k) & \ldots & u_{\mathrm{t}}\left(k+N_{p}-1\right)
\end{array}\right]^{T} \\
\tilde{P}_{d}(k)=\left[\begin{array}{lll}
P_{d}(k) & \ldots & P_{d}\left(k+N_{p}-1\right)
\end{array}\right]^{T} .
\end{gathered}
$$

The variable $u_{\mathrm{t}}(k)$ represents the total power generation of fuel cell cars at time step $k: u_{\mathrm{t}}(k)=\sum_{i \in \mathcal{I}} u_{f, i}(k)$.

With a given value for $\lambda(k)$, the minimization part of (21) can be separated, i.e., distributed, between the cars. For fuel cell car number $i \in \mathcal{I}$, the following problem should be solved:

$$
\begin{aligned}
\tilde{V}_{i}^{*}(k)=\underset{\tilde{V}_{i}(k)}{\arg \min } & \left(\tilde{V}_{i}^{T}(k) W_{q, i}(k) \tilde{V}_{i}(k)+W_{v, i}(k) \tilde{V}_{i}(k)\right. \\
& \left.\left.+W_{d, i}(k) \hat{w}_{p, i}(k)\right)+\lambda^{T}(k) \tilde{u}_{f, i}(k)\right)
\end{aligned}
$$

subject to

$$
\begin{gathered}
G_{1, i}\left(\hat{w}_{1, i}(k)\right) \tilde{V}_{i}(k) \leq G_{2, i}(k)+G_{3, i}(k) x_{i}(k) \\
\vdots \\
G_{1, i}\left(\hat{w}_{N, i}(k)\right) \tilde{V}_{i}(k) \leq G_{2, i}(k)+G_{3, i}(k) x_{i}(k) .
\end{gathered}
$$

All the optimization variables in (22) belong to a single car and the problem can be solved without any dependencies on other fuel cell cars. The solution of (22) and (23), $\tilde{V}_{i}^{*}(k)$, is in fact a function of $\lambda(k)$, where $\lambda(k+j)$ for $j \in \mathcal{P}$ can be interpreted as a signal to determine the need for power generation in different time steps. A lower value for $\lambda(k)$ indicates more need for power generation at time step $k$. The maximization part of (21) is called the master problem and can be written in the form of

$$
\max _{\lambda(k) \in \mathbb{R}^{N_{p}}} \sum_{i \in \mathcal{I}} \tilde{V}_{i}^{*}(\lambda(k)) .
$$

Note that $\sum_{i \in \mathcal{I}} \tilde{V}_{i}^{*}(\lambda(k))$ is a concave function of $\lambda(k)$, because it is the minimum of a collection of linear functions in $\lambda(k)$. Therefore, (24) can be solved by a gradient ascent algorithm. We consider a coordinator to solve the master problem (24). Note that only the variables related to the power generation, $u_{f, i}(k+j)$, for $i \in \mathcal{I}$ and $j \in \mathcal{P}$, are involved in the master problem (24) and, hence, driving patterns of the cars, i.e., $\lambda_{f, i}(k+j)$, for $i \in \mathcal{I}$ and $j \in \mathcal{P}$, are not required in solving the master problem. Therefore, no privacy-sensitive data on usage of the cars need to be shared between the fuel cell cars and a central coordinator.

\section{B. ADMM Method}

In the method of ADMM, the dual problem is constructed based on an augmented Lagrangian function, $L_{\rho}$ [25]. By considering the definition of the cost function in (7) and the power 
balance constraint, the augmented Lagrangian function is of the form

$$
\begin{aligned}
L_{\rho}(\tilde{u}(k), \tilde{z}(k), \lambda)= & J(k)+\lambda^{T}\left(\tilde{u}_{\mathrm{t}}(k)-\tilde{P}_{d}(k)\right) \\
& +\frac{\rho}{2}\left\|\tilde{u}_{\mathrm{t}}(k)-\tilde{P}_{d}(k)\right\|_{2}^{2}
\end{aligned}
$$

where $\rho$ is a penalty parameter. The dual problem is then

$$
\begin{aligned}
& \text { D : } \max _{\lambda \in \mathbb{R}^{N_{p}}} \min _{\tilde{u}(k), \tilde{z}(k)} L_{\rho}(\tilde{u}(k), \tilde{z}(k), \lambda) \\
& \text { s.t. (14). }
\end{aligned}
$$

Based on the ADMM approach, in order to solve (26), each fuel cell requires to solve the following optimization problem:

$$
\begin{aligned}
& \min _{\tilde{u}_{i}(k), \tilde{z}_{i}(k)} L_{\rho, i}\left(\tilde{u}_{i}(k), \tilde{z}_{i}(k), \tilde{u}_{r}(k), \lambda\right) \\
& \text { s.t. }(23)
\end{aligned}
$$

where $L_{\rho, i}$ and $\tilde{u}_{r}(k)$ are defined as follows:

$$
\begin{aligned}
L_{\rho, i}(k, \lambda)= & J_{i}(k)+\lambda^{T}\left(\tilde{u}_{f, i}(k)+\tilde{u}_{r}(k)-\tilde{P}_{d}(k)\right) \\
& +\frac{\rho}{2}\left\|\tilde{u}_{f, i}(k)+\tilde{u}_{r}(k)-\tilde{P}_{d}(k)\right\|_{2}^{2} \\
\tilde{u}_{r}(k)= & {\left[\begin{array}{lll}
u_{r}(k) & \ldots & u_{r}\left(k+N_{p}-1\right)
\end{array}\right]^{T} . }
\end{aligned}
$$

In the above-mentioned definition, $J_{i}(k)$ is a part of the cost function (6) related to fuel cell $i$ and $u_{r}(k)$ is the total power generation of all the fuel cells except fuel cell $i$ at time step $k$ as follows:

$$
\begin{gathered}
J_{i}(k)=\sum_{j \in \mathcal{P}}\left(W_{p, i} u_{f, i}^{2}(k+j)+W_{s, i}\left|\Delta s_{f, i}(k+j)\right|\right. \\
\left.+C_{\mathrm{e}}(k+j) w_{i}(k+j)\right) \\
u_{r}(k)=\sum_{n \in \mathcal{I}} u_{f, n}(k)-u_{f, i}(k) .
\end{gathered}
$$

If the value of $\tilde{u}_{r}(k)$ is known for fuel cell $i,(27)$ can be written as an MIQP problem

$$
\begin{aligned}
& \min _{\tilde{V}_{i}(k)} \tilde{V}_{i}^{T}(k) M_{q, i}(k) \tilde{V}_{i}(k)+M_{v, i}(k) \tilde{V}_{i}(k) \\
& \text { s.t. (23) }
\end{aligned}
$$

where $M_{q, i}(k)$ and $M_{v, i}(k)$ can be determined based on the model and driving pattern of fuel cell car $i$.

In the ADMM method, different agents solve a minimization problem one after another. In fact, each agent minimizes the augmented Lagrangian function by assuming that all the other agents have already made their decision. The determined solution, or the decision of the agent, will be shared with the next agent and the same procedure will be repeated until the last agent. After solving the last optimization problem, the coordinator will be informed about the decisions of all the agents. The coordinator executes a gradient ascent algorithm, and as a result, new values for the Lagrangian multipliers will be determined. The coordinator propagates these updated values among all the agents. In our problem formulation, only the power generation profiles of fuel cells are involved in the global constraint. Therefore, there is no need to share all the optimization variables with other agents. It is worth mentioning that the driving patterns of the cars can also be kept private, as they are not directly involved in the global constraint. The interested reader is referred to [25] for a review of the ADMM algorithm.

\section{PADMM Method}

In the PADMM [26], the coordinator and all the cars are following the same procedure as described in Section IV-B. However, the optimization problem that is solved in the fuel cell cars is different because a proximal term is added to the objective function. Therefore, rather than solving (28), fuel cell $i$ solves the following problem during each iteration at time step $k$ :

$$
\begin{aligned}
& \min _{\tilde{V}_{i}(k)} \tilde{V}_{i}^{T}(k) M_{q, i}(k) \tilde{V}_{i}(k)+M_{v, i}(k) \tilde{V}_{i}(k) \\
& \quad+\frac{1}{2}\left(\tilde{V}_{i}(k)-V_{i}^{\text {prev }}(k)\right)^{T} Q_{i}\left(\tilde{V}_{i}(k)-V_{i}^{\text {prev }}(k)\right) \\
& \text { s.t. (23) }
\end{aligned}
$$

where $V_{i}^{\text {prev }}(k)$ indicates the vector of optimization variables related to agent $i$ determined at the previous iteration. At the first iteration, we assume that $V_{i}^{\text {prev }}(k)=0$. The matrix $Q_{i}$ is a weight factor. The purpose of adding the proximal term to the objective function of each agent is to achieve a faster convergence compared to the ADMM method [26], [27]. Note that (29) is still an MIQP problem.

\section{Discussion on Data Exchange}

All the methods developed in this section are designed according to a distributed architecture, therewith guaranteeing the privacy of the cars' driving patterns, and are able to handle uncertainty in the power generation of the fuel cells. During each iteration of the dual decomposition method, the coordinator propagates the value of $\lambda(k)$ to all the fuel cell cars and each fuel cell car $i \in \mathcal{I}$ sends back its decision regarding $\tilde{u}_{f, i}(k)$ to the coordinator. Therefore, the information that is exchanged at each iteration consists of $2 N_{p} N_{\text {veh }}$ variables. A communication channel between the coordinator and fuel cell cars suffices to implement the dual decomposition method. For the ADMM and PADMM methods, the value of $\tilde{u}_{\mathrm{t}}(k)$ should be transferred from one fuel cell car to its nearest neighbor. Therefore, the communication network topology required by the ADMM and PADMM methods is different from the one required by the dual decomposition method in the sense that individual fuel cell cars should be able to communicate with their adjacent fuel cell cars. In the ADMM and PADMM methods, the total information exchanged at each iteration is $N_{p}\left(2 N_{\text {veh }}+1\right)$ variables, which is close to the total information exchanged using the dual decomposition method.

Note that a typical sample time for power scheduling in such systems is between 10 and $15 \mathrm{~min}$. Therefore, the available time for determining control inputs at each sample time is at least $10 \mathrm{~min}$. As a result, the communication process time is negligible in this type of application.

\section{Illustrative Case Studies}

In this section, the results of simulating the developed control methods in different microgrids are reported. The total number of fuel cell cars, $N_{\mathrm{veh}}$, in the microgrid 


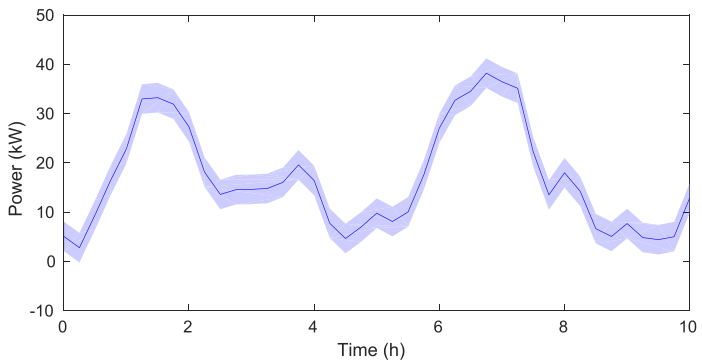

Fig. 1. Residual load of the microgrid, $P_{d}(k)$, with four fuel cell cars. The solid line indicates the predicted value, while the actual residual load will be realized inside the shaded area.

is variable among different simulation scenarios. In line with [20], the characteristics of fuel cell $i$ are given by $\alpha_{f, i}=$ $0.05+0.001 i+\gamma_{\alpha, i}$ and $\beta_{f, i}=0.09+0.0001 i+\gamma_{\beta, i} ;$ so note that the characteristics are different for each fuel cell. The parameters $\gamma_{\alpha, i}$ and $\gamma_{\beta, i}$ represent the difference between the simulation model of the plant and the model that is used in the controller. Their values are set to zero in the control model, while in the simulation, we selected random real numbers in the range $[-0.001,0.001]$ for $\gamma_{\alpha, i}$ and $[-0.002,0.002]$ for $\gamma_{\beta, i}$. The maximum power generation of any fuel cell, $\bar{u}_{f, i}$, is assumed to be $15 \mathrm{~kW}$ and the fuel tank of any car has a capacity $\bar{x}_{f, i}$ of $5 \mathrm{~kg}$ for all $i \in \mathcal{I}$. In order to preserve some fuel for transportation purposes, we consider $\underline{x}_{f, i}=2$ $\mathrm{kg}$ as the minimum level of fuel in each car. The time interval between the two consecutive time steps, $T_{\mathrm{S}}$, is $15 \mathrm{~min}$.

The values of $W_{p, i}$ and $W_{s, i}$ in the cost function (6) are $0.5+0.005 i$ and $1.5+0.005 i$, respectively. We consider a number of cars, $N_{\mathrm{veh}}$, that vary from 4 to 50 . Following the standard tuning rule in MPC [28], we have selected the prediction horizon in such a way that during the prediction horizon window, the most important dynamics of the system can be covered. For example, $N_{p}=6$ can cover the refilling process and power generation of a car in the future. Any prediction horizon around this number is suitable for our application and we used $N_{p}=4,6$, and 8 to show that the improvement of the performance by using DF MM approach is not dependent on the specific selection of the control horizon.

The upper and lower bounds on the uncertainty of power generation of each fuel cell are assumed to have equal magnitude, but different signs, i.e., $\bar{w}_{i}=-\underline{w}_{i}$. The value of $\bar{w}_{i}$ varies from 0.5 to $2 \mathrm{~kW}$ for all $i \in \mathcal{I}$ in different simulations, but we assume that in each simulation, $\bar{w}_{i}$ is the same for all $i \in \mathcal{I}$. Fig. 1 depicts the residual load, $P_{d}(k)$, of a microgrid that contains four fuel cell cars during the $10 \mathrm{~h}$ of our simulation. In the rest of our case studies, the residual load will increase in direct proportion to the number of cars. In order to obtain a comparison between the systems with different sizes, we consider that the fuel cell cars do not leave the microgrid. The refilling rate of all cars, $R_{f, i}$ for all $i$, is considered to be $2 \mathrm{~kg}$ per time step.

The value of 0.1 is selected for the penalty parameter, $\rho$, in the ADMM and PADMM methods. In order to solve the optimization problems of the PADMM method fast, the weight factor $Q_{i}$ is determined to have a diagonal form
TABLE II

Operational Cost of a Microgrid With Centralized ARCHITECTURE USING THE DF MM APPROACH AND THE MM APPROACH

\begin{tabular}{|c|c|c|c|c|c|c|}
\hline$N_{\mathrm{p}}$ & \multicolumn{2}{|c|}{4} & \multicolumn{2}{c|}{6} & \multicolumn{2}{c|}{8} \\
\hline $\bar{w}_{i}$ & MM & DF & MM & DF & MM & DF \\
\hline 0.5 & 2064.20 & 1818.91 & 2082.39 & 1876.21 & 2079.55 & 1900.61 \\
\hline 1 & 2068.44 & 1761.74 & 2086.53 & 1754.12 & 2084.05 & 1851.52 \\
\hline 1.5 & 2094.26 & 1698.76 & 2098.83 & 1508.34 & 2093.41 & 1482.97 \\
\hline 2 & 2114.67 & 1453.71 & 2119.25 & 1511.26 & 2114.81 & 1552.50 \\
\hline
\end{tabular}

$Q_{i}=\operatorname{diag}\left\{W_{s, i} \mathbf{I}_{3 N_{\text {veh }} \times 3 N_{\text {veh }}}, \mathbf{0}_{N_{\text {veh }} \times N_{\text {veh }}}\right\}$ for the MM method. In the DF MM method, the structure of $Q_{i}$ remains the same, while the size of the diagonal blocks changes with respect to the changes in the size of $\tilde{u}_{i}(k)$ and $\tilde{z}_{i}(k)$.

Table II lists the performance of the two developed centralized approaches, i.e., the MM approach and the DF MM approach, for a microgrid containing four fuel cell cars. The results show that for different values of the prediction horizon, $N_{p}$, and the disturbance bound, $\bar{w}_{i}$, the DF MM approach outperforms the MM approach. These results are as expected, because the level of conservatism in the DF approach is less than the MM approach.

In order to compare the performance of the three distributed control approaches, the system is first simulated with a central control system. Then, the operational cost of each distributed control approach is compared to the centralized solution in order to determine a measure for the loss of performance induced by the distributed solution. For example, if the operational cost of the system using the dual decomposition system is determined by $J_{\mathrm{dd}}$ and the centralized MPC cost is $J_{c}$, we define the performance loss, $e_{\mathrm{dd}}$, as follows:

$$
e_{\mathrm{dd}} \triangleq\left|\frac{J_{\mathrm{dd}}-J_{c}}{J_{c}}\right| \times 100 \% \text {. }
$$

A lower value for $e_{\mathrm{dd}}$ indicates that the performance of the dual decomposition approach is closer to the centralized solution. Similarly, we define two other measures for the performance loss, $e_{\text {admm }}$ and $e_{\text {padmm }}$, related to the ADMM and PADMM approaches. Fig. 2 depicts the performance loss of each distributed control method when the number of fuel cell cars is changing, for $N_{p}=6$, and $\bar{w}_{i}=1$. For the ADMM and PADMM methods, the performance loss drops significantly when the number of fuel cell cars in the system increases. In fact, when the number of fuel cell cars in the system increases, the influence of a single fuel cell car on the total power generation decreases. Therefore, the influence of binary variables $s_{f, i}$ and $s_{r, i}$ for $i \in \mathcal{I}$ on the total power generation decreases when the number of fuel cell cars in the system increases. As a result, the optimization problem becomes closer to a convex programming case, where the solution of primal and dual problems is identical. This fact explains the decrease of performance loss in the ADMM and PADMM when the number of fuel cell cars in the system increases. For a system with a small number of fuel cell cars, compared to the other distributed methods using the DF MM 


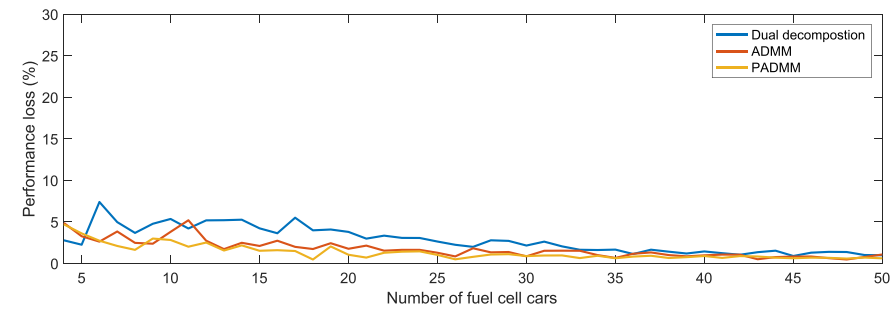

(a)

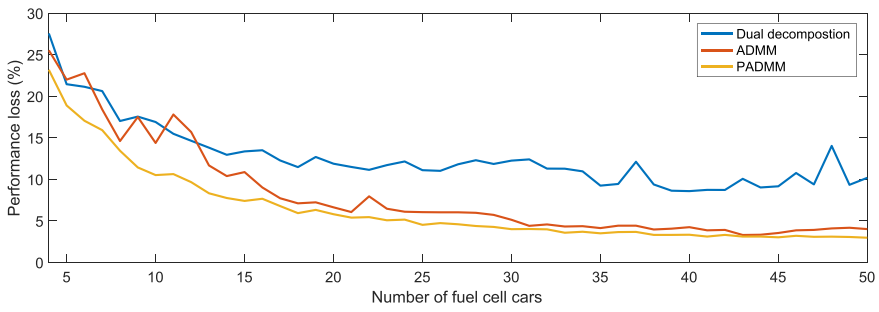

(b)

Fig. 2. Performance loss of distributed control systems, $e_{\mathrm{dd}}, e_{\mathrm{admm}}$, and $e_{\text {padmm }}$, with respect to the number of fuel cell cars inside the system for (a) MM approach and (b) DF MM approach.

approach, the performance of PADMM is the closest to the one of the centralized solution.

In general, the reason for the performance loss in all the distributed methods is that in some time steps, the power balance is achieved by using the auxiliary generator/batteries. In the distributed control architectures, two types of power generators/storage are used. The first type is the fleet of fuel cell cars and the second type is auxiliary generator/batteries. In the case that the power balance is not maintained by the fuel cell car, the auxiliary generator/battery is used to maintain the power balance. As this auxiliary equipment is much more expensive to operate compared to fuel cell cars, in the case that there is a mismatch between the power generation of fuel cell cars and the residual load of the microgrid, the power balance is maintained by using the auxiliary equipment and, hence, the operational cost increases. The large performance loss of dual decomposition method is mainly the result of a relatively high level of power imbalance after scheduling the power generation profile of all the fuel cell cars.

In order to show the satisfactory performance of the distributed ADMM and PADMM approaches when the number of fuel cell cars is high enough, we have simulated different scenarios by considering different levels of the disturbance and different values for the prediction horizon. Due to the finite horizon of our MPC methods, there is not a known theoretical relation between the value of the parameters $N_{p}$ and $\bar{\omega}_{i}$ and the actual closed-loop system performance. Therefore, we have simulated the system for different values of $N_{p}$ and $\bar{\omega}_{i}$, and as we can check from Tables III and IV, for different values of $N_{p}$ and $\bar{\omega}_{i}$, the performance loss of the two distributed approaches with respect to the centralized approach remains small.

In the two centralized control methods, i.e., the centralized MM method and the centralized DF MM method, the driving patterns of the cars are shared with a centralized controller.
TABLE III

Percentage of Performance Loss of ADMM and PADMM in a Microgrid With 50 FUel CELl CARS AND FOR DifFERENT VALUES OF $N_{p}$ AND $\bar{w}$, USING THE MM APPROACH

\begin{tabular}{|c|c|c|c|c|c|c|}
\hline$N_{\mathrm{p}}$ & \multicolumn{2}{|c|}{4} & \multicolumn{2}{c|}{6} & \multicolumn{2}{c|}{8} \\
\hline $\bar{w}_{i}$ & $e_{\text {admm }}$ & $e_{\text {padmm }}$ & $e_{\text {admm }}$ & $e_{\text {padmm }}$ & $e_{\text {admm }}$ & $e_{\text {padmm }}$ \\
\hline 0.5 & 0.02 & 0.4 & 0.03 & 0.61 & 0.04 & 0.40 \\
\hline 1 & 0.68 & 0.48 & 1.02 & 0.57 & 1.64 & 0.49 \\
\hline 1.5 & 0.52 & 0.64 & 1.26 & 0.78 & 1.8 & 1.06 \\
\hline 2 & 1.2 & 0.93 & 1.94 & 1.05 & 2.87 & 1.09 \\
\hline
\end{tabular}

TABLE IV

Percentage of Performance Loss of ADMM and PADMM in a Microgrid With 50 Fuel CEll CARS AND For DifFERENT VALUES OF $N_{p}$ AND $\bar{w}$, Using THE DF MM APPROACH

\begin{tabular}{|c|c|c|c|c|c|c|}
\hline$N_{\mathrm{p}}$ & \multicolumn{2}{|c|}{4} & \multicolumn{2}{c|}{6} & \multicolumn{2}{c|}{8} \\
\hline $\bar{w}_{i}$ & $e_{\text {admm }}$ & $e_{\text {padmm }}$ & $e_{\text {admm }}$ & $e_{\text {padmm }}$ & $e_{\text {admm }}$ & $e_{\text {padmm }}$ \\
\hline 0.5 & 10.26 & 1.12 & 5.93 & 1.35 & 4.56 & 1.55 \\
\hline 1 & 4.03 & 2.66 & 3.53 & 2.63 & 4.57 & 3.49 \\
\hline 1.5 & 3.53 & 2.67 & 5.05 & 3.89 & 4.97 & 3.68 \\
\hline 2 & 6.42 & 5.25 & 5.35 & 4.24 & 7.07 & 5.45 \\
\hline
\end{tabular}

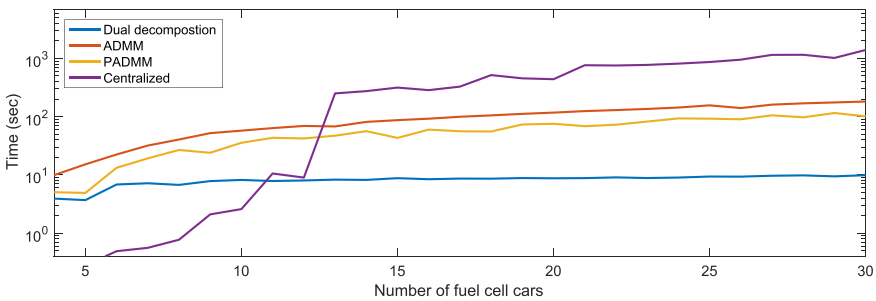

(a)

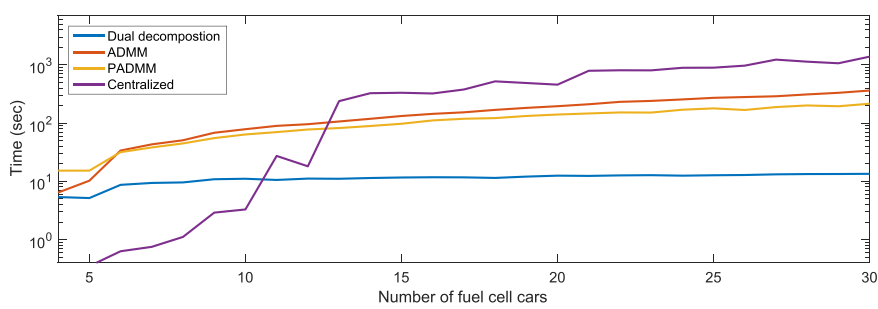

(b)

Fig. 3. Computation time of different control strategies with respect to the number of fuel cell cars using (a) MM approach and (b) DF MM approach.

In the rest of the methods, i.e., all the distributed methods, the driving patterns are kept private. Fig. 2 demonstrates a comparison between the centralized methods, i.e., the methods that the driving patterns have to be shared, and the distributed methods, i.e., the methods that keep the driving patterns private. In general, the centralized methods have the best performance, because the controller can determine the optimal control input. In the distributed methods, the performance decreases. However, Fig. 2 indicates that for a system with more than 30 fuel cell cars, the performance loss of the ADMM and PADMM methods is negligible.

Fig. 3 shows a comparison between the optimization time of different control strategies. From this figure, we can conclude that the optimization problems of distributed control methods can be solved significantly faster than those of the centralized methods. Here, the communication process between different 
TABLE V

Computation Time (in Seconds) of DifFEREnt Control Strategies FOR A Microgrid With 50 FuEl CEll CARS, $\bar{w}_{i}=0.5$, AND FOR DIFFERENT VALUES OF $N_{p}$, USING THE MM AND THE DF MM APPROACHES

\begin{tabular}{|c|c|c|c|}
\hline Method & 4 & 6 & 8 \\
\hline \hline Centralized MM & 1214.67 & 1608.43 & 2105.31 \\
\hline Dual Decomposition MM & 6.22 & 10.99 & 18.63 \\
\hline ADMM MM & 187.55 & 346.65 & 607.10 \\
\hline PADMM MM & 77.47 & 110.75 & 214.27 \\
\hline Centralized DF & 1237.07 & 1620.16 & 2105.75 \\
\hline Dual Decomposition DF & 8.59 & 15.26 & 25.06 \\
\hline ADMM DF & 421.05 & 701.53 & 1083.42 \\
\hline PADMM DF & 188.58 & 300.80 & 503.46 \\
\hline
\end{tabular}

agents and between each agent and the coordinator is not taken into account and the sole purpose of Fig. 3 is the comparison in the computation time required to solve the optimization problems. All the simulations are run on a Linux machine with $16 \mathrm{~GB}$ of RAM and an Intel Xeon CPU with eight cores and 3.7-GHz clock speed. The solver Gurobi is used in all the simulations. Note that the optimization problems of different cars are solved in parallel in the dual decomposition approach. As a result, increasing the number of cars does not influence the computation time of this method. We have used a serial implementation of the ADMM and PADMM methods, see [27]. In a serial implementation of the ADMM, agents solve their respective optimization problems consecutively. In other words, the optimization problems are solved serially and the result is that increasing the number of agents in the system increases the total computation time. However, compared to the centralized control architecture, ADMM and PADMM methods still require much less computation time. In order to prevent the shortage of memory, we have set a limit on the computation time of each optimization problem. In our simulations, the computation times of the distributed methods were all below this limit. However, in the case of the centralized architecture for large number of cars, this limit was reached and thus acted as a hard constraint for the solver. The reason that the computation time of the centralized control architecture in Fig. 3 does not grow exponentially after around 10 fuel cell cars is, in fact, the limit that is set in the solver. Table $\mathrm{V}$ demonstrates the computation time of different control methods by using different prediction horizons.

\section{CONCLUSION}

A fleet of fuel cell cars can be considered as a distributed power generation system inside an islanded mode microgrid and an appropriate power scheduling of the fuel cells can maintain the power balance of the microgrid. We have shown that the MM and the DF MM methods are able to schedule the power generation of fuel cell cars by minimizing the operational cost of the system with respect to the physical and operational cost of the system. Both of the control methods can deal with uncertainty in the prediction of the residual load of the microgrid. A centralized architecture to implement MM and DF MM methods is disadvantageous because the privacy of the car owner, with respect to driving patterns, cannot be respected in such a centralized setting. However, in the proposed distributed control methods, the driving pattern of any car is kept private and not shared with any other agent. Another advantage of the proposed distributed methods is their scalability and applicability in large-scale systems by reducing the computational burden of the solution of the optimization problems. The mentioned advantages of the distributed control methods come with the cost of losing a certain level of performance in the operation of the system. However, case studies show that the performance loss is related to the number of fuel cell cars in the system and becomes smaller for large vehicle numbers, which would be the case in envisioned practical scenarios. Among the three distributed control methods, the PADMM has the least loss of performance when the number of cars is small. When the number of cars in the system grows, the performance of the ADMM and the PADMM methods become closer to the performance of a centralized control architecture.

Topics for future research include the simulation of microgrid while considering the network topology and the model of the low-level controllers, the development of a distributed control architecture for the operation of a microgrid in the grid-connected mode, clustering the fuel cell cars in the ADMM method, and adjusting the current control methods to deal with more complex models of the system.

\section{REFERENCES}

[1] J. A. P. Lopes, C. L. Moreira, and A. G. Madureira, "Defining control strategies for microgrids islanded operation," IEEE Trans. Power Syst., vol. 21, no. 2, pp. 916-924, May 2006.

[2] V. Oldenbroek, L. A. Verhoef, and A. J. M. van Wijk, "Fuel cell electric vehicle as a power plant: Fully renewable integrated transport and energy system design and analysis for smart city areas," Int. J. Hydrogen Energy, vol. 42, no. 12, pp. 8166-8196, Mar. 2017.

[3] J. A. Moncada, E. P. Lee, G. D. C. N. Guerrero, O. Okur, S. T. Chakraborty, and Z. Lukszo, "Complex systems engineering: Designing in sociotechnical systems for the energy transition," EAI Endorsed Trans. Energy Web, vol. 17, no. 11, Jul. 2017.

[4] A. J. M. van Wijk and L. Verhoef, Our Car as Power Plant. Delft, The Netherlands: Delft Univ. Press, 2014.

[5] V. Puig, A. Rosich, C. Ocampo-Martinez, and R. Sarrate, "Fault-tolerant explicit MPC of PEM fuel cells," in Proc. 46th IEEE Conf. Decision Control, New Orleans, LA, USA, Dec. 2007, pp. 2657-2662.

[6] J. Schiffer, R. Ortega, A. Astolfi, J. Raisch, and T. Sezi, "Conditions for stability of droop-controlled inverter-based microgrids," Automatica, vol. 50, no. 10, pp. 2457-2469, Oct. 2014.

[7] J. Schiffer, D. Zonetti, R. Ortega, A. M. Stanković, T. Sezi, and J. Raisch, "A survey on modeling of microgrids-From fundamental physics to phasors and voltage sources," Automatica, vol. 74, pp. 135-150, Dec. 2016.

[8] C. Kammer and A. Karimi, "Decentralized and distributed transient control for microgrids," IEEE Trans. Control Syst. Technol., vol. 27, no. 1, pp. 311-322, Jan. 2019.

[9] J. M. Guerrero, J. C. Vasquez, J. Mata, L. G. de Vicuña, and M. Castilla, "Hierarchical control of droop-controlled AC and DC microgridsA general approach toward standardization," IEEE Trans. Ind. Electron. vol. 58, no. 1, pp. 158-172, Jan. 2011.

[10] A. Parisio, E. Rikos, and L. Glielmo, "A model predictive control approach to microgrid operation optimization," IEEE Trans. Control Syst. Technol., vol. 22, no. 5, pp. 1813-1827, Sep. 2014.

[11] I. Prodan and E. Zio, "A model predictive control framework for reliable microgrid energy management," Int. J. Elect. Power Energy Syst., vol. 61, pp. 399-409, Oct. 2014.

[12] P. M. Namara, R. R. Negenborn, B. De Schutter, and G. Lightbody, "Optimal coordination of a multiple HVDC link system using centralized and distributed control," IEEE Trans. Control Syst. Technol., vol. 21, no. 2, pp. 302-314, Mar. 2013 
[13] C. A. Hans, V. Nenchev, J. Raisch, and C. Reincke-Collon, "Minimax model predictive operation control of microgrids," IFAC Proc. Volumes, vol. 47, no. 3, pp. 10287-10292, Aug. 2014.

[14] A. Parisio and L. Glielmo, "Stochastic model predictive control for economic/environmental operation management of microgrids," in Proc. Eur. Control Conf., Zurich, Switzerland, Jul. 2013, pp. 2014-2019.

[15] S. R. Cominesi, M. Farina, L. Giulioni, B. Picasso, and R. Scattolini, "A two-layer stochastic model predictive control scheme for microgrids," IEEE Trans. Control Syst. Technol., vol. 26, no. 1, pp. 1-13, Jan. 2018.

[16] T. Borsche, F. Oldewurtel, and G. Andersson, "Scenario-based MPC for energy schedule compliance with demand response," IFAC Proc. Volumes, vol. 47, no. 3, pp. 10299-10304, Aug. 2014.

[17] F. Valencia, D. Sáez, J. Collado, F. Ávila, A. Marquez, an J. J. Espinosa, "Robust energy management system based on interval fuzzy models," IEEE Trans. Control Syst. Technol., vol. 24, no. 1, pp. 140-157, Jan. 2016

[18] F. Alavi, N. van de Wouw, and B. De Schutter, "Power scheduling in islanded-mode microgrids using fuel cell vehicles," in Proc. IEEE 56th Annu. Conf. Decision Control, Melbourne, VIC, Australia, Dec. 2017, pp. 5056-5061.

[19] F. Alavi, E. P. Lee, N. van de Wouw, B. De Schutter, and Z. Lukszo, "Fuel cell cars in a microgrid for synergies between hydrogen and electricity networks," Appl. Energy, vol. 192, pp. 296-304, Apr. 2017.

[20] P. Rodatz, G. Paganelli, A. Sciarretta, and L. Guzzella, "Optimal power management of an experimental fuel cell/supercapacitor-powered hybrid vehicle," Control Eng. Pract., vol. 13, no. 1, pp. 41-53, Jan. 2005.

[21] A. Bemporad and M. Morari, "Control of systems integrating logic, dynamics, and constraints," Automatica, vol. 35, no. 3, pp. 407-427, Mar. 1999.

[22] G. Gross and F. D. Galiana, "Short-term load forecasting," Proc. IEEE, vol. 75, no. 12, pp. 1558-1573, Dec. 1987.

[23] Gurobi Optimization. Accessed: Jan. 10, 2019. [Online]. Available: http://www.gurobi.com/

[24] D. P. Bertsekas, Nonlinear Programming. Belmont, MA, USA: Athena Scientific, 1999

[25] S. Boyd, N. Parikh, E. Chu, B. Peleato, and J. Eckstein, "Distributed optimization and statistical learning via the alternating direction method of multipliers," Found. Trends Mach. Learn., vol. 3, no. 1, pp. 1-122, Jul. 2011.

[26] J. Eckstein, "Some saddle-function splitting methods for convex programming," Optim. Methods Softw., vol. 4, no. 1, pp. 75-83, Mar. 1994.

[27] J. J. Wang and W. Song, "An algorithm twisted from generalized ADMM for multi-block separable convex minimization models," J. Comput. Appl. Math., vol. 309, pp. 342-358, Jan. 2017.

[28] J. L. Garriga and M. Soroush, "Model predictive control tuning methods: A review," Ind. Eng. Chem. Res., vol. 49, no. 8, pp. 3505-3515, Mar. 2010

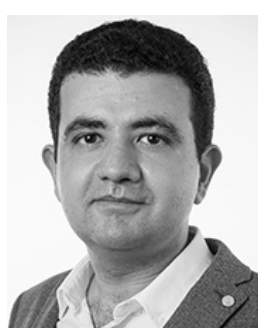

Farid Alavi received the B.Sc. and M.Sc. degrees in electrical engineering-control from the Isfahan University of Technology, Isfahan, Iran, in 2010 and 2013, respectively, and the Ph.D. degree from the Delft University of Technology, Delft, The Netherlands, in 2019. His Ph.D. research with the Delft Center for Systems and Control was on model predictive control (MPC) of energy management systems in the presence of uncertainty.

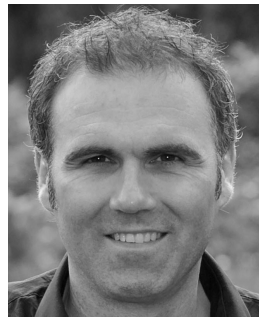

Nathan van de Wouw received the M.Sc. degree (Hons.) and the Ph.D. degree in mechanical engineering from the Eindhoven University of Technology, Eindhoven, The Netherlands, in 1994 and 1999, respectively.

He was with Philips Applied Technologies, Eindhoven, in 2000, and the Netherlands Organisation for Applied Scientific Research, Delft, The Netherlands, in 2001. He was a Visiting Professor with the University of California at Santa Barbara, Santa Barbara, CA, USA, in 2006 and 2007, the University of Melbourne, Melbourne, Australia, in 2009 and 2010, and the University of Minnesota, Minneapolis, MN, USA, in 2012 and 2013. From 2015 to 2019, he was a part-time Full Professor with the Delft University of Technology, Delft. He is currently a Full Professor with the Mechanical Engineering Department, Eindhoven University of Technology. He also holds an Adjunct Full Professor with the University of Minnesota.

Dr. van de Wouw was a recipient of the IEEE Control Systems Technology Award for the development and application of variable-gain control techniques for high-performance motion systems in 2015 .

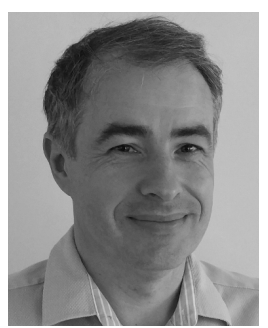

networks. INTELLIGENT TRANSPORTATION SYSTEMS and an Associate Editor of the IEEE TRAnsactions on Automatic Control. 